\title{
Association of unilateral lattice corneal dystrophy on slit lamp and bilateral confocal microscopy features with H572R mutation in the TGFBI gene
}

\author{
Ricardo De Sousa Peixoto ${ }^{1}$ Stacey Mutch ${ }^{2} \cdot$ Jacqueline Eason $^{3} \cdot$ Kaie Jaakson ${ }^{4} \cdot$ Eneli Haamer $^{4}$. \\ Veerabahu Senthil Maharajan ${ }^{1}$
}

Received: 29 November 2018 / Revised: 24 May 2019 / Accepted: 12 June 2019 / Published online: 3 July 2019

(c) The Royal College of Ophthalmologists 2019

Lattice corneal dystrophy (LCD) type 1 is a progressive dystrophy characterised by accumulation of amyloid in the corneal stroma, which has the appearance of thin branching reflective lights, leading to deterioration of visual acuity [1]. Lattice dystrophy can be identified by histology with Congo red, green birefringence with polarising filter and by confocal microscopy (IVCM) [1]. Unilateral lattice dystrophy (ULD) cases have been reported in the literature [2-5] associated with different pathogenic variances $[3,5]$, including the $p$. (His572del), which was suggested to cause unilateral presentation [3].

We previously reported one case of seemingly ULD, where a 24-year-old patient (patient 1) had clinical signs only in the left eye (Fig. 1a, b) but displayed evidence of LCD bilaterally with IVCM (Fig. 2a, b). Similarly, a 41year-old patient (patient 2) was referred with slit lamp signs in the right eye only (Fig. 1c, d) but had bilateral lattice evidence with IVCM (Fig. 2c, d). This patient developed early lattice changes in the previously asymptomatic eye 5 years after first seen (Fig. 1e, f). A 31-year-old patient (patient 3) presented with unilateral dystrophy on slit lamp biomicroscopy (Fig. 1g, h) but was found to have bilateral lattice deposits with confocal

Veerabahu Senthil Maharajan

senthil.maharajan@nuh.nhs.uk

1 Ophthalmology department, Nottingham University Hospitals trust, Nottingham NG7 2UH, UK

2 East Midlands Regional Molecular Genetics Laboratory, Nottingham University Hospitals trust, Nottingham NG5 1PB, UK

3 Clinical Genetics Service, Nottingham University Hospitals trust, Nottingham NG5 1PB, UK

4 Asper Biogene LLC, Vaksali 17a, 50410 Tartu, Estonia microscopy (Fig. 2e, f). Genetic testing was performed in two patients (patients 1 and 2), who were both found to have a heterozygous mutation in exon $13 \mathrm{c} .1715 \mathrm{~A}>\mathrm{G}$ p. (His572Arg), changing histidine to arginine at codon 572 (H572R). The third patient did not consent to have genetic testing.

Our findings provide further evidence that asymptomatic eyes in ULD may have sub-clinical disease, and that in early stages ULD can only be identified by IVCM, which is necessary for correct diagnosis. We also show that subclinical disease may develop into symptomatic disease in the long term and advise that patients need to be counselled that their unaffected eye may develop disease.

This rare asymmetric presentation was associated with H572R mutation in the two patients who agreed to have genetic testing. A mutation at this amino acid was previously reported with late unilateral presentation (at 63 years old) but IVCM was not performed and sub-clinical presentation was not excluded [3]. This mutation has also been linked with bilateral disease in patients where presentation was in the 3rd and 4th decades [5]. The three patients in this study are older than the classical 1st decade presentation for LCD. Our findings, taken together with those in literature, suggest that mutations affecting this amino acid may lead to a less aggressive phenotype, including asymmetric and asynchronous presentation, and that onset may be later than with classic LCD. We suggest that restraint should be exercised with diagnosing unilateral corneal lattice until changes have been excluded in IVCM. We also suggest that unless proven otherwise, unilateral LCD should be called asymmetrical LCD. This may have significant implications on patient's expectations and advice given.

Acknowledgements We would like to thank Mouhamed Al-Aqaba for help with processing and analysis of figures. 
Fig. 1 Slit lamp photographs. Arrows indicate presence of lattice lines. a, b belong to patient 1, who has the H572R mutation. a belongs to right eye (asymptomatic) and $\mathbf{b}$ belongs to left eye (symptomatic). c, d, e and $\mathbf{f}$ belong to patient 2, who has the H572R mutation. c, d were taken at referral. c shows right eye (symptomatic) and $\mathbf{d}$ shows left eye (asymptomatic). e, $\mathbf{f}$ show left eye 5 years after referral. e indicates an area of mild lattice lines, which are more distinguishable on retroillumination, as shown in $\mathbf{f}$ $\mathbf{g}, \mathbf{h}$ belong to patient 3 , who declined genetic analysis. $\mathbf{g}$ belongs to the right eye (asymptomatic) and $\mathbf{h}$ to the symptomatic left eye

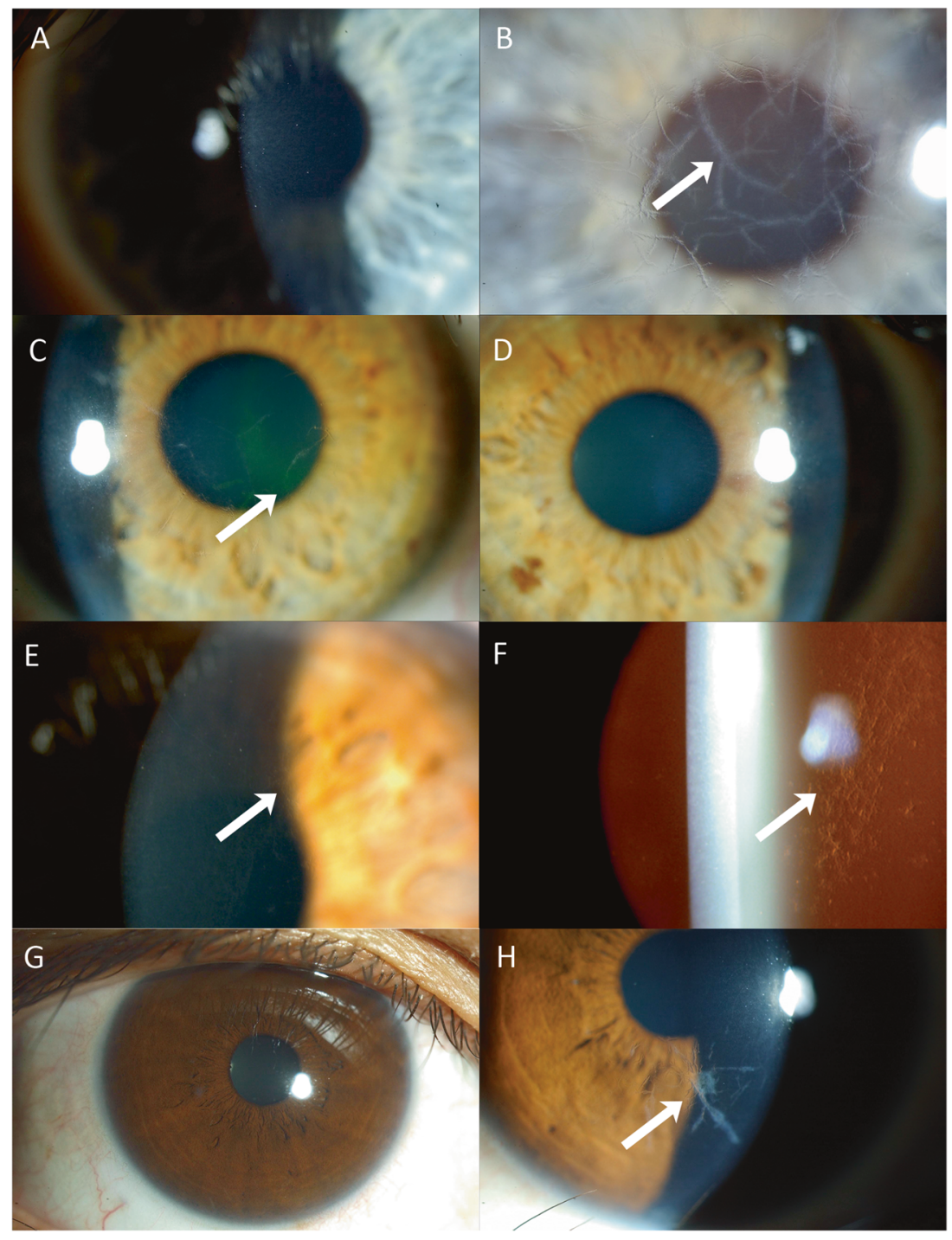


Fig. 2 IVCM photographs. Arrows indicate presence of amyloid deposits. a, b belong to patient 1 . a corneal stroma (65 $\mu \mathrm{m}$ depth) of the unaffected right eye. b corneal stromal section $(75 \mu \mathrm{m}$ depth) of the affected left eye. c, $\mathbf{d}$ belong to patient 2 at referral. c corneal stroma $(100 \mu \mathrm{m}$ depth) of the affected right eye. $\mathbf{d}$ corneal stroma $(106 \mu \mathrm{m}$ depth) of the unaffected left eye. $\mathbf{e}, \mathbf{f}$ belong to patient 3. e corneal stroma (201 $\mu \mathrm{m}$ depth) of the unaffected right eye. f corneal stroma (47 $\mu \mathrm{m}$ depth) of the affected left eye
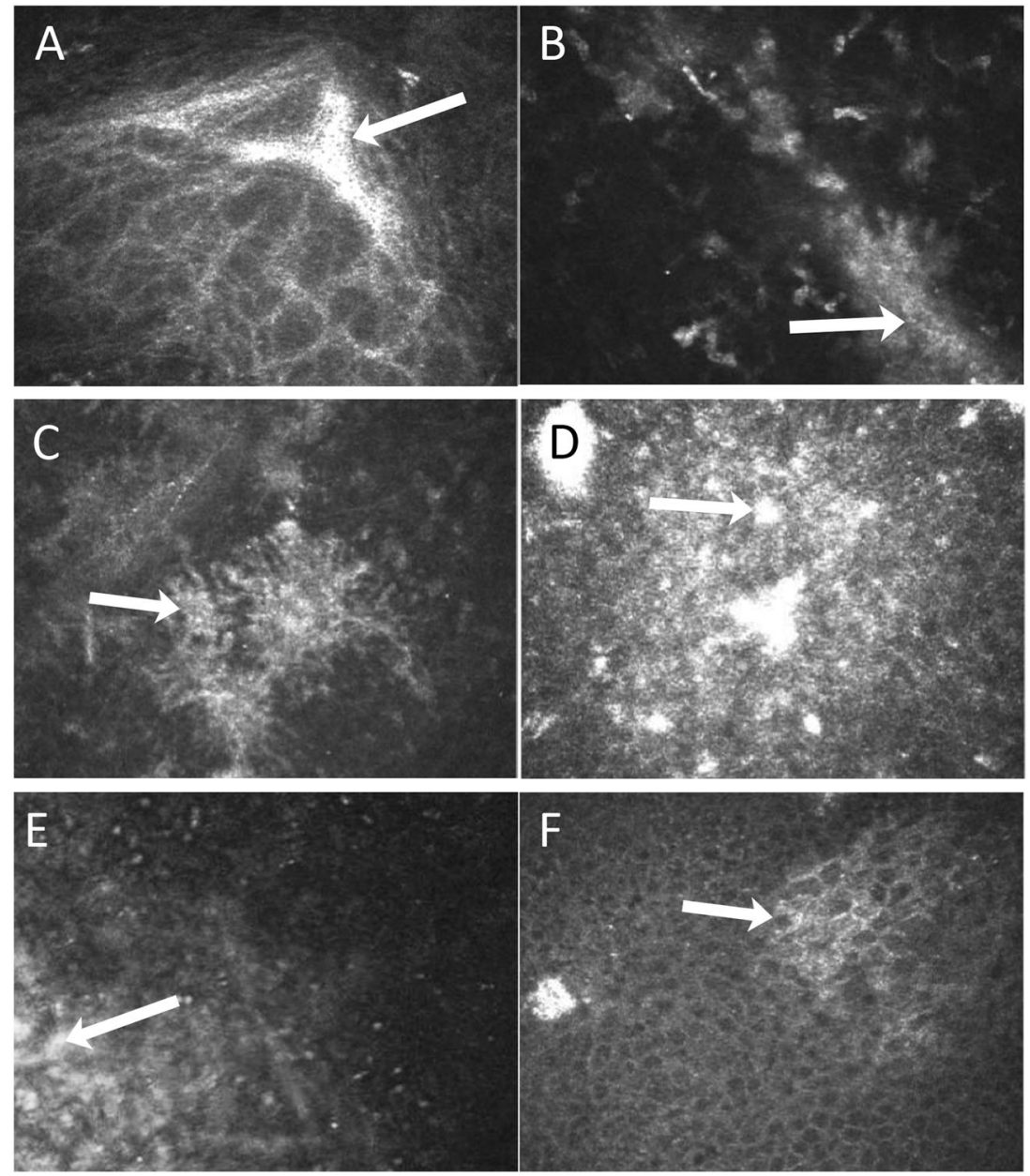

\section{Compliance with ethical standards}

Conflict of interest The authors declare that they have no conflict of interest.

Publisher's note: Springer Nature remains neutral with regard to jurisdictional claims in published maps and institutional affiliations.

\section{References}

1. Kobayashi A, Fujiki K, Fujimaki T, Murakami A, Sugiyama K. In vivo laser confocal microscopic findings of corneal stromal dystrophies. Arch Ophthalmol. 2007;125:1168-73.
2. Kailasanathan A, Maharajan S. In vivo confocal microscopy detects preclinical Lattice corneal dystrophy. Eye. 2013;27:991-2.

3. Aldave AJ, Rayner SA, Kim BT, Prechanond A, Yellore VS. Unilateral lattice corneal dystrophy associated with the novel His572del mutation in the TGFBI gene. Mol Vis. 2006;27:1.

4. Kojima Y, Inoue T, Hori Y, Maeda N, Nishida K. Unilateral variant of late-onset lattice corneal dystrophy with the Pro501Thr mutation in the TGFBI gene without deposits in the unaffected cornea using confocal microscopy. Cornea. 2013;32: 1396-8.

5. Atchaneeyasakul LO, Appukuttan B, Pingsuthiwong S, Yenchitsomanus PT, Trinavarat A, Srisawat C, Study Group. A novel H572R mutation in the transforming growth factor-beta-induced gene in a Thai family with lattice corneal dystrophy type I. Jpn J Ophthalmol. 2006;50:403-8. 